\title{
CT features of novel coronavirus pneumonia (COVID-19) in children
}

\author{
Ya-ni Duan ${ }^{1} \cdot$ Yan-qiu Zhu ${ }^{1} \cdot$ Lei-lei Tang ${ }^{1} \cdot$ Jie Qin $^{1}$
}

Received: 12 March 2020 / Revised: 28 March 2020 / Accepted: 1 April 2020 / Published online: 14 April 2020

(C) European Society of Radiology 2020

\begin{abstract}
A serious epidemic of COVID-19 broke out in Wuhan, Hubei Province, China, and spread to other Chinese cities and several countries now. As the majority of patients infected with COVID-19 had chest CT abnormality, chest CT has become an important tool for early diagnosis of COVID-19 and monitoring disease progression. There is growing evidence that children are also susceptible to COVID-19 and have atypical presentations compared with adults. This review is mainly about the differences in clinical symptom spectrum, diagnosis of COVID-19, and CT imaging findings between adults and children, while highlighting the value of radiology in prevention and control of COVID-19 in pediatric patients.

\section{Key points}

- Compared with adults, pediatric patients with COVID-19 have the characteristics of lower incidence, slighter clinical symptoms, shorter course of disease, and fewer severe cases.

- The chest CT characteristics of COVID-19 in pediatric patients were atypical, with more localized GGO extent, lower GGO attenuation, and relatively rare interlobular septal thickening.

- Chest CT should be used with more caution in pediatric patients with COVID-19 to protect this vulnerable population from risking radiation.
\end{abstract}

Keywords COVID-19 · Pneumonia, viral · Tomography, spiral computed

$\begin{array}{ll}\begin{array}{l}\text { Abbreviations } \\ \text { 2019-nCoV }\end{array} & \begin{array}{l}\text { 2019 Novel coronavirus } \\ \text { COVID-19 }\end{array} \\ \text { Coronavirus disease 2019 } \\ \text { GGO } & \begin{array}{l}\text { Ground glass opacity } \\ \text { Multiple organ dysfunction syndrome } \\ \text { MODS }\end{array} \\ \text { RT-PCR } & \begin{array}{l}\text { Reverse transcription-polymerase } \\ \text { chain reaction }\end{array} \\ \text { SARS } & \begin{array}{l}\text { Severe Acute Respiratory Syndrome } \\ \text { Severe Acute Respiratory } \\ \text { SARS-CoV-2 }\end{array} \\ & \text { Syndrome Coronavirus 2 }\end{array}$

\section{Introduction}

In December 2019, a mysterious pneumonia broke out in Wuhan and then spread to other Chinese cities and several

Jie Qin

jason020@163.com

1 Department of Radiology, The 3rd Affiliated Hospital of Sun Yat-sen University-Lingnan Hospital, 2693 Kaichuang Avenue, Guangzhou 510760, People's Republic of China countries [1]. On January 7, 2020, a new type of coronavirus was successfully isolated by the Chinese Center for Disease Control and Prevention [2]. On January 12, 2020, the WHO named the virus 2019-nCoV temporarily. On February 11, 2020, the International Committee on Taxonomy of Viruses (ICTV) proposed to name the new virus SARS-CoV-2 due to its similarity with SARS occurred in 2003 [3]. On the same day, the WHO named the disease caused by SARS-CoV-2 infection COVID-19 [4]. Since the outbreak of COVID-19, the Chinese government has taken various measures to stop epidemic. As of today, 16 weeks after the onset of epidemic, China's domestic COVID-19 epidemic has been well controlled, and the daily increase of confirmed cases in China mainland dropped to zero for the first time on March 18, 2020 [5]. However, the virus outbreak in countries outside China is on the rise; Europe and America are severely affected at this moment, which means that COVID-19 has evolved from an epidemic to pandemic. Countries should take COVID-19 threat seriously and make a joint effort to fight against the disease.

In the early days of the outbreak, the first reported 425 laboratory-confirmed COVID-19 patients in Wuhan before January 22, 2020, showed that there were no cases in children under the age of 15 [6]. Soon afterwards, another 1099 
laboratory-diagnosed cases from all over China through January 29,2020 , indicated that $0.9 \%$ of patients were aged below 15 years, which means that COVID-19 can be spread within the whole age spectrum [7]. As of February 11, 2020, 965 people aged 19 years or younger were laboratorydiagnosed with COVID-19, accounting for $2 \%$ of all cases ( 965 of 44,672) [8]. One of the more recent epidemiological studies of COVID-19 in Shenzhen, China, revealed that the infection rate in children under $10(7.4 \%)$ was similar to the population average (7.9\%) [9]. According to published reports, the first diagnosed pediatric patient was observed on January 20, 2020. Besides, a 14-year-old boy died on February 7, 2020 [10]. The youngest infected person tested positive just minutes after birth in London [11]. Most pediatric patients were mild, with much fewer severe and critical cases (5.9\%) than adult patients (18.5\%) [10]. Up to now, fewer studies reported possible vertical transmission of SARS$\mathrm{CoV}-2$ as IgG was detected positive in newborns of infected mothers $[12,13]$.

Limited by the high false negative rates and kit supply, RTPCR cannot give a satisfied performance for diagnosing suspicious patients as much as possible [14]. The total positive rate of RT-PCR of throat swabs was reported to be about 30$60 \%$ at initial testing [15]. According to $\mathrm{Ai} \mathrm{T}$ et al, by taking RT-PCR results as reference, the sensitivity, specificity, and accuracy of chest CT in COVID-19 diagnosis were 97\%, $25 \%$, and $68 \%$, respectively [16]. Chest CT can detect lung abnormalities before RT-PCR assay turning up positive, even in those asymptomatic carriers $[17,18]$. For this reason, chest CT abnormalities have been incorporated into the clinically diagnosed criteria from February 12, 2020, in Hubei province, but removed from the sixth version of guidelines on February 19, 2020, possibly due to low specificity. Compared with adults, pediatric patients have slighter clinical symptoms, which strengthens the importance of chest $\mathrm{CT}$ in the early diagnosis of COVID-19 in pediatric patients [17]. In this review, we summarized the difference in clinical symptom spectrum, diagnosis of COVID-19 infection, and particularly CT imaging findings between adults and children.

\section{Clinical symptom spectrum}

The clinical symptoms of pediatric patients are non-specific. In the initial stage, fever and cough are most commonly seen. Besides, other symptoms might appear to be nasal congestion, runny nose, sore throat, fatigue, headache, myalgia, vomiting, and diarrhea $[11,19,20]$. Among the pediatric patients, most of them had mild or moderate fever generally lasting 1-2 days $[11,19]$. Specifically, in some cases, gastrointestinal symptoms could be main symptoms and even initial symptoms [21, 22]. Also, it might be hard to notice the symptoms among infants [19]. Some distinct symptoms of children such as irritability, decreased response, and poor feeding could be the sign of infection [22]. Some patients were asymptomatic [17, 23]. In addition, leucocytes and lymphocytes might decrease and infection-related biomarkers might increase. However, compared with adults, pediatric patients were more likely to have above laboratory data within the normal range $[11,17,24,25]$. On the whole, the majority of the cases were mild or moderate types with recovery within a week [11, 17]. Nevertheless, a few pediatric patients deteriorated in about a week, which could be companied with dyspnea, cyanosis, progressive lymphocyte reduction, platelet decrease, transaminase or creatine kinase increase, etc. Later, the disease would develop progressively into acute respiratory distress syndrome (ARDS), respiratory failure, septic shock, multiple organ dysfunction syndrome (MODS), and coagulation disorders [19, 21]. It was more possible for pediatric patients with chronic medical illness and young children, particularly infants, to deteriorate severely. But the proportion of critical type in pediatric patients was smaller than in adult patients $[11,19,24,26]$. A 14-year-old boy from Hubei province died on February 7 , 2020 [10]. In a word, compared with adult patients, pediatric patients had milder symptoms, quicker recovery, and better prognosis $[1,11]$.

\section{Diagnosis of COVID-19 infection}

The criteria for diagnosis of COVID-19 in children were formulated based on the diagnosis of adult patients, combined with the characteristics of epidemiology and clinical manifestations of children. Similar to adults, epidemiological history should be confirmed first. It takes one exposure history and two clinical manifestations to diagnosis as suspected case (Table 1) [19]. Since most pediatric patients, whose symptoms were nonspecific and mild, connected with cluster outbreaks and these days are in flu season, researchers divided epidemiological history into different risk levels, based on which they establish targeted diagnostic criteria with emphasis on the exclusion of other pathogens that might cause similar symptoms. Therefore, the improvement allows children with high exposure risk to be diagnosed earlier and the range of suspected cases to be more accurately divided (Table 1) [11]. In general, positive real-time fluorescence polymerase chain reaction of the patient's respiratory or blood specimen for SARS-CoV-2 nucleic acid is needed, but some confirmed cases could get negative results [19,21]. Furthermore, confirmed cases could be divided into 5 types according to respective clinical manifestations (Table 2) [11].

Now that COVID-19-related chest CT abnormalities could be prior to clinical symptoms [18] or could comparatively be more severe with milder symptoms [27] and could appear in patients with negative RT-PCR for COVID-19 [28], chest CT might have good potential in screening and early diagnosis of COVID-19. Moreover, it was reported that the sensitivity of chest CT was greater than that of RT-PCR, which supported 
Table 1 Two versions of recommendations for the diagnosis of COVID-19 in children

Diagnosis of COVID-19 in Diagnosis and treatment recommendation for pediatric children
Recommendations for the diagnosis, prevention, and control of the 2019 novel coronavirus infection in children (first interim edition)

Epidemiological history

Surveillance cases Suspected
cases

Confirmed cases

(1) Travel or residence history in Wuhan or other areas with recent local transmission within 2 weeks before onset;

(2) Close contact with people with fever or respiratory symptoms from Wuhan or other areas with recent local transmission within 2 weeks before onset;

(3) Close contact with confirmed or suspected cases of COVID-19 within 2 weeks before onset;

(4) Cluster outbreaks: Besides this child, there are other patients with fever or respiratory symptoms, including suspected or confirmed cases of COVID-19;

(5) Neonate with suspected or confirmed COVID-19 pregnant mother.

Clinical manifestations for diagnosis of suspected cases

Diagnosis of suspected cases
(1) Fever, fatigue, dry cough. Some patients could be symptomatic or have low-grade fever;

(2) Lung imaging showed SARS-CoV-2 pneumonia signs;

(3) In the early stage, leucocytes reduced or be within the normal range or lymphocytes decreased.

One exposure history and two clinical manifestations

Suspected cases have 1 of the following etiology test results:

(1) Positive real-time fluorescence polymerase chain reaction of pharyngeal swab, sputum, stool, or blood specimen for SARS-CoV-2 nucleic acid;

(2) Virus gene sequencing founded virus that is highly homologous with the known SARS-CoV-2, contained in above specimens;

(3) SARS-CoV-2 particles were isolated and cultured from the above specimens.
Epidemiological classification:

(1) High risk: close contact with confirmed or suspected cases of COVID-19 within 14 days before onset;

(2) Medium risk: cluster outbreaks of COVID-19 in the place of residence or community;

(3) Low risk: no cluster outbreaks in the community of residence and general endemic areas outside the source of the epidemic.

(1) Asymptomatic children at high risk;

(2) Children at medium or low risk have one of the following symptoms:

1) Fever;

2) Respiratory symptoms, fatigue, nausea, vomiting, abdominal discomfort, diarrhea, etc.

(1) Fever persisted, obvious respiratory symptoms, shortness of breath or decreased pulse oxygen saturation, or gastrointestinal manifestations such as nausea, vomiting, abdominal discomfort and diarrhea;

(2) Laboratory tests: leucocytes reduced or did not, lymphocyte decrease, CRP slightly elevated or did not;

(3) Lung imaging showed SARS-CoV-2 pneumonia signs.

(1) Neonate with confirmed COVID-19 postpartum women;

(2) Children at high risk have 2 of the clinical manifestations;

(3) Surveillance cases at medium or low risk have 2 of the clinical manifestations after exclusion of flu (regular administration of oseltamivir phosphate for 2 days made no effect) and other common pathogen infections.

Suspected cases had positive real-time fluorescence polymerase chain reaction of pharyngeal swab, sputum, stool, or blood specimen for SARS-CoV-2 nucleic acid or founded virus that is highly homologous with the known SARS-CoV-2, contained in above specimens, by virus gene sequencing. the use of CT for screening and diagnosis of patients with characteristics of epidemiology and clinical manifestations of COVID-19 and negative RT-PCR [29]. Meanwhile, the National Health Commission of the People's Republic of China recommended that any suspected cases with COVID19-related CT scan results should be counted as clinically diagnosed cases.

\section{CT imaging findings}

To date, several studies [17, 30-36] and a few case reports [21-23, 37-45] have explored chest CT signs of COVID-19 in children and newborns. The CT manifestations of COVID-19 in pediatric patients are diverse and lack specificity. Some mild pediatric patients with COVID-19 show normal findings 
Table 2 Clinical classification of COVID-19 in children

\begin{tabular}{|c|c|}
\hline $\begin{array}{l}\text { Clinical } \\
\text { classification }\end{array}$ & Clinical manifestations \\
\hline Asymptomatic & $\begin{array}{l}\text { No clinical signs and symptoms, lung imaging was normal, but SARS-CoV-2 nucleic acid } \\
\text { test was positive, or positive serum-specific antibody which helped retrospective } \\
\text { diagnosis of infection. }\end{array}$ \\
\hline Mild & $\begin{array}{l}\text { The clinical symptoms are mild, with symptoms of upper respiratory tract infections, } \\
\text { including fever, cough, and sore throat, or symptoms of gastrointestinal symptoms such } \\
\text { as nausea, vomiting, abdominal pain, and diarrhea, without sign of pneumonia. }\end{array}$ \\
\hline Moderate & $\begin{array}{l}\text { It could have typical COVID-19 manifestations. Fever and cough were common. In the } \\
\text { initial stage, dry cough appeared mostly, followed by sputum cough. Some might have } \\
\text { wheezing without obvious hypoxia such as shortness of breath, dry rales and/or wet rales. } \\
\text { Chest imaging showed changes of pneumonia. Some children had no clinical signs and } \\
\text { symptoms, but chest CT showed lung lesions, which were subclinical. }\end{array}$ \\
\hline \multirow[t]{6}{*}{ Severe } & $\begin{array}{l}\text { The disease usually progresses in about } 1 \text { week and has at least one of the following } \\
\text { conditions: }\end{array}$ \\
\hline & $\begin{array}{l}\text { (1) Respiratory rate increased (RR): Children under } 1 \text { year of age, } R R \geq 70 / \mathrm{min} \text {, above } \\
1 \text { year of age } R R \geq 50 / \mathrm{min} \text {, with exclusion of the impact of fever and crying; }\end{array}$ \\
\hline & (2) Blood oxygen saturation at rest $<92 \%$; \\
\hline & $\begin{array}{l}\text { (3) With symptoms indicated hypoxia: assisted breathing (groaning, wing flaps, sags, etc.), } \\
\text { cyanosis, intermittent apnea; }\end{array}$ \\
\hline & (4) Unconsciousness: lethargy, coma, convulsions; \\
\hline & (5) Refused to eat or had poor feeding, had signs of dehydration. \\
\hline \multirow[t]{4}{*}{ Critical } & The disease progresses rapidly and have at least one of the following conditions: \\
\hline & (1) Respiratory failure occurs and requires mechanical ventilation; \\
\hline & (2) Shock; \\
\hline & (3) Combined with other organ failures, requires intensive care unit. \\
\hline
\end{tabular}

on chest CT [17, 30-33, 41]. In those patients presenting lung abnormality, ground glass opacity (GGO) mainly in the peripheral and posterior lungs that did not spare the subpleural regions is the most frequently observed performance [17, 30-33]. Compared with adults, GGO in pediatric patients with COVID-19 has the characteristics of more localized extent, lower attenuation, and less lobular involvement (Fig. 1). Feng $\mathrm{K}$ et al reported that small nodular GGO may be a suggestive appearance of pediatric patients with epidemiology [17]. Moreover, other manifestations such as consolidation, GGO with consolidation, or interlobular septa thickening can also be observed in the pediatric patients [30, 31, 33]. We summarized the chest CT imaging characteristics of pediatric patients that have been reported so far in Table 3 . Although milder patients are more common in children, a few of them may turn into disease progression. In these patients, GGO may develop into multiple consolidation with the extent of GGO enlarged and attenuation of GGO increased $[21,30]$. Moreover, the lung interstitial changes may become more obvious than before. In very rare cases, diffuse lung consolidation occurs, presenting "white lung" [30]. In resolving stage, lung lesions will be completely resolved or only remain minimal linear opacities [37]. In addition to the CT findings mentioned above, it is worth noting that in some cases the high spatial resolution $\mathrm{CT}$ changes are similar to those of bronchopneumonia, manifesting as a patchy opacity along the bronchial vascular bundle. Meanwhile, one of these cases presenting as bronchopneumonia complicate with mycoplasma infection [30]. As Xia et al reported, underlying coinfection is very common in pediatric patients ( 9 of $20,45 \%$ ), which means that case exclusion should be more carefully done while pediatric patients have definite epidemiological history but atypical CT findings [33]. Pleural effusion was reported in several pediatric patients $[31,34,36]$. No cases reported lymphadenopathy.

\section{The value of radiology in prevention and control of COVID-19}

Although chest CT has been recommended as one of optional criteria for the clinical diagnosis of COVID-19 in pediatric patients [19], it must be admitted that fewer pediatric patients have typical imaging features than adult patients, which may lead to missed diagnosis if only relying on chest $\mathrm{CT}$ to screen pediatric patients. In those highly suspected cases but initial RT-PCR results show negative, chest CT may provide support information for diagnosis and management, but final diagnosis still rely on further nucleic acid detection. 

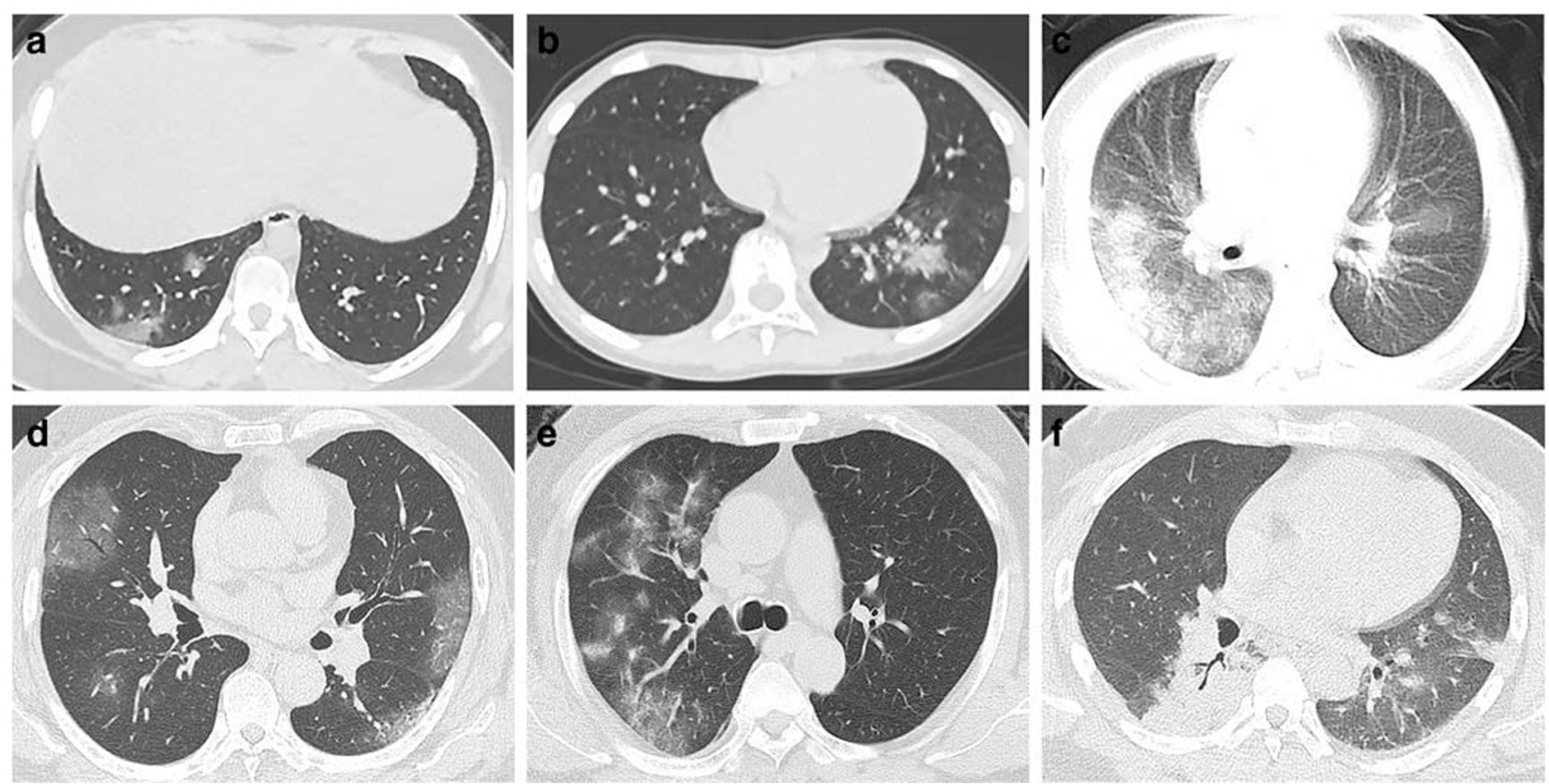

Fig. 1 Chest CT imaging of coronavirus disease 2019 (COVID-19) pneumonia in children and adults. a Female, 14 years old. Chest CT showed scattered GGO in the inferior lobe of the right lung, located subpleural or extended from subpleural lesions. b Male, 10 years old. Chest CT showed consolidation with halo sign in the inferior lobe of the left lung surrounded by GGO. c Male, 1 year old. Chest CT showed diffused consolidations and GGO in both lungs, with a "white lung"

appearance of the right lung. d Male, 49 years old. Chest CT showed multiple subpleural GGO in both lungs. e Male, 64 years old. Chest CT showed multiple GGO and consolidations in the right upper lobe. $\mathbf{f ~ M a l e , ~}$ 34 years old. Chest CT showed diffused consolidation in the right lower lobe and left lung with fewer GGO surrounded. * Fig. 1 a to $\mathrm{c}$ is reproduced with permission from Xia W et al. [33]

Moreover, there are some pediatric patients with RT-PCR positively showing normal findings on chest $\mathrm{CT}[17,30,32$, $33,41]$, perhaps due to a relatively high proportion of mild and moderate types. Although some of negative-CT pediatric

patients with COVID-19 can present abnormality in follow-up CT (perhaps that infection is in the earliest stage at the time of initial chest CT performing) [17], these slight imaging changes are not enough to affect clinical decisions in mild and

Table 3 Initial chest CT imaging features of coronavirus disease 2019 (COVID-19) pneumonia in children

\begin{tabular}{|c|c|c|c|c|c|c|}
\hline & Normal & GGO & GGO + consolidation & GGO + interlobular septal thickening & Consolidation & Others \\
\hline Ma YL et $\mathrm{al}^{\#}(n=115)$ & 27 & \multicolumn{4}{|c|}{ No detailed classification in other 49 cases } & $39^{\mathrm{a}}$ \\
\hline Wang $\mathrm{D}$ et al* $(n=31)$ & 17 & 9 & 1 & / & / & $2^{\mathrm{a}, \mathrm{b}}$ \\
\hline Ma HJ et al $(n=22)$ & 2 & 6 & 6 & 2 & 3 & $3^{\mathrm{b}}$ \\
\hline Xia W et al $(n=20)$ & 4 & 12 & 10 & 4 & & $3^{\mathrm{c}}$ \\
\hline Feng K et al $(n=15)$ & 6 & 9 & l & l & l & / \\
\hline Zhong $\mathrm{Z}$ et al $(n=9)$ & 4 & 4 & l & l & 1 & / \\
\hline Zhou YY et al $(n=9)$ & 1 & 1 & 6 & l & 1 & 0 \\
\hline Case reports ${ }^{\Psi}(n=17)$ & 4 & 4 & 6 & / & 1 & $2^{\mathrm{a}, \mathrm{d}}$ \\
\hline
\end{tabular}

${ }^{\text {a }}$ Increased bronchovascular shadows

${ }^{\mathrm{b}}$ Similar to bronchopneumonia

${ }^{\mathrm{c}}$ Tiny nodules

${ }^{\mathrm{d}}$ Scattered small strip-like opacities

${ }^{\#}$ Lung CT findings showed ground glass opacity, fiber opacities, patchy changes, and pulmonary consolidation in 49 children lacking classification, among whom 2 children had "white lung"

${ }^{\Psi}$ Case reports include refs. $21-23,37-45$

*No detailed CT imaging description in another two atypical patients

$G G O$ ground glass opacity 
moderate type of pediatric patients, which limited the value of follow-up CT. According to the Guideline for Medical Imaging in Auxiliary Diagnosis of COVID-19, low-dose CT scan should be performed on infant patients and the follow-up imaging should be reduced as less frequently as possible [37]. Thus, for radiation protection considerations, follow-up imaging is necessary only in cases of clinical deterioration for pediatric patients. If patients need follow-up, chest radiograph might be a better alternative. They will not be able to show the ground glass abnormalities as we see on CT, but it may be less important since these patients will not be as sick as patients presenting with consolidation.

Comparing the current guideline for COVID-19 and recommendations specific for pediatric patients with COVID-19, the common contents of the release criteria include no fever for 3 days, significantly improved respiratory symptoms, and negative nucleic acid test for two consecutive sputum, nasopharyngeal swab, or other respiratory tract specimens (sampling time at least $24 \mathrm{~h}$ apart). But in pediatric patients, exudative inflammation resolved apparently in lung imaging is not necessary for discharging from hospital $[19,46]$.

When SARS-CoV-2 co-infected with other pathogen infections, abnormalities on chest $\mathrm{CT}$ may not follow the same course as clinical findings and take longer to resolve. Therefore, chest CT examination must be chosen with extreme caution for protecting the vulnerable population from unnecessary radiation risk. Detection of common clinical pathogen and further nucleic acid test for various source samples are needed in guiding diagnosis and treatment. Good clinical indications are more than ever important during treatment.

\section{Conclusion}

Compared with those in adults, the chest CT characteristics of COVID-19 in children were atypical, with more localized GGO extent, lower GGO attenuation, and relatively rare interlobular septal thickening. Most pediatric patients are mild type, which requires a balance between the risk of radiation and necessity for chest CT. If necessary, low-dose CT scan is more suitable for this population. Follow-up imaging is only necessary in cases of clinical deterioration and should be reduced as less frequently as possible. Good clinical indications are important more than ever, in order to not spread the disease in the hospital by transferring patients from wards to $\mathrm{CT}$ and for radiation protection of this vulnerable patient group. In conclusion, pediatric patients with COVID-19 commonly have lighter lung abnormalities than adults, and chest CT should be used with more caution in pediatric patients.

Funding information This study has received funding from the National Natural Science Foundation of China (grant number 81101096); the Medical Scientific Research Foundation of Guangdong Province (grant number B2011102); Science and Technology Planning Project of Guangdong Province (grant number 2015A020212017); and the Natural Science Foundation of Guangdong Province (grant numbers 2016A030313323 and 2017A030313841).

\section{Compliance with ethical standards}

Guarantor The scientific guarantor of this publication is Jie Qin (jason020@163.com).

Conflict of interest The authors of this manuscript declare no relationships with any companies, whose products or services may be related to the subject matter of the article.

Statistics and biometry Not applicable for a review.

Informed consent Not applicable for a review.

Ethical approval This review was approved by the Institutional Review Board of the Third Affiliated Hospital of Sun Yat-sen University, Guangzhou, China.

\section{Methodology}

- Literature review

\section{References}

1. Huang C, Wang Y, Li X et al (2020) Clinical features of patients infected with 2019 novel coronavirus in Wuhan, China. Lancet 395: 497-506

2. Zhu N, Zhang D, Wang W et al (2020) A novel coronavirus from patients with pneumonia in China, 2019. N Engl J Med 382:727-733

3. Gorbalenya AE, Baker SC, Baric RS et al (2020) Severe acute respiratory syndrome-related coronavirus: the species and its viruses - a statement of the Coronavirus Study Group. bioRxiv. https:// doi.org/10.1101/2020.02.07.937862

4. World Health Organization (2020) WHO Director-General's remarks at the media briefing on 2019-nCoV on 11 February 2020. World Health Organization, Geneva. Available via https://www. who.int/dg/speeches/detail/who-director-general-s-remarks-at-themedia-briefing-on-2019-ncov-on-11-february-2020. Accessed 10 Apr 2020

5. National Health Commission of the People's Republic of China (2020) Update on COVID-19 as of 24:00 March 18. National Health Commission of the People's Republic of China, China. Available via http://www.nhc.gov.cn/xcs/yqtb/202003/ e644c2fc18b4448db7ed4b30f68b91a6.shtml. Accessed 19 Mar 2020 (in Chinese)

6. Li Q, Guan X, Wu P et al (2020) Early transmission dynamics in Wuhan, China, of novel coronavirus-infected pneumonia. N Engl J Med 382:1199-1207

7. Guan W, Ni Z, Hu Y et al (2020) Clinical characteristics of 2019 novel coronavirus infection in China. N Engl J Med. https://oi.org/ 10.1056/NEJMoa2002032

8. Wu Z, McGoogan JM (2020) Characteristics of and important lessons from the coronavirus disease 2019 (COVID-19) outbreak in China: summary of a report of 72314 cases from the Chinese Center for Disease Control and Prevention. JAMA. https://doi.org/10. 1001/jama.2020.2648

9. Bi Q, Wu Y, Mei S et al (2020) Epidemiology and transmission of COVID-19 in Shenzhen China: analysis of 391 cases and 1,286 of 
their close contacts. medRxiv. https://doi.org/10.1101/2020.03.03. 20028423

10. Dong Y, Mo X, Hu Y et al (2020) Epidemiology of COVID-19 Among Children in China. Pediatrics. https://doi.org/10.1542/ peds.2020-0702

11. Society of Pediatrics, Chinese Medical Association; Editorial Board, Chinese Journal of Pediatrics (2020) Recommendations for the diagnosis, prevention and control of the 2019 novel coronavirus infection in children (first interim edition). Zhonghua Er Ke Za Zhi 58:169-174 (in Chinese)

12. Dong L, Tian J, He S et al (2020) Possible vertical transmission of SARS-CoV-2 from an infected mother to her newborn. JAMA. https://doi.org/10.1001/jama.2020.4621

13. Zeng $\mathrm{H}, \mathrm{Xu} \mathrm{C}$, Fan $\mathrm{J}$ et al (2020) Antibodies in infants born to mothers with COVID-19 pneumonia. JAMA. https://doi.org/10. 1001/jama.2020.4861

14. Li YY, Wang WN, Lei Y et al (2020) Comparison of the clinical characteristics between RNA positive and negative patients clinically diagnosed with 2019 novel coronavirus pneumonia. Zhonghua Jie He He Hu Xi Za Zhi 43:E023 (in Chinese)

15. Yang Y, Yang M, Shen C et al (2020) Evaluating the accuracy of different respiratory specimens in the laboratory diagnosis and monitoring the viral shedding of $2019-\mathrm{nCoV}$ infections. medRxiv. https://doi.org/10.1101/2020.02.11.20021493

16. Ai T, Yang Z, Hou H et al (2020) Correlation of chest CT and RTPCR testing in coronavirus disease 2019 (COVID-19) in China: a report of 1014 cases. Radiology. https://doi.org/10.1148/radiol. 2020200642

17. Feng K, Yun YX, Wang XF et al (2020) Analysis of CT features of 15 children with 2019 novel coronavirus infection. Zhonghua Er Ke Za Zhi 58:E007 (in Chinese)

18. Pan Y, Guan H, Zhou S et al (2020) Initial CT findings and temporal changes in patients with the novel coronavirus pneumonia (2019nCoV): a study of 63 patients in Wuhan, China. Eur Radiol. https:// doi.org/10.1007/s00330-020-06731-x

19. Chen Z, Fu J, Shu Q et al (2020) Diagnosis and treatment recommendation for pediatric coronavirus disease-19. Zhejiang Da Xue Xue Bao Yi Xue Ban 49:1-8 (in Chinese)

20. Wei M, Yuan J, Liu Y, Fu T, Yu X, Zhang ZJ (2020) Novel coronavirus infection in hospitalized infants under 1 year of age in China. JAMA. https://doi.org/10.1001/jama.2020.2131

21. Chen F, Liu ZS, Zhang FR et al (2020) First case of severe childhood novel coronavirus pneumonia in China. Zhonghua Er Ke Za Zhi 58:E005 (in Chinese)

22. Zeng LK, Tao XW, Yuan WH, Wang J, Liu X, Liu ZS (2020) First case of neonate infected with novel coronavirus pneumonia in China. Zhonghua Er Ke Za Zhi 58:E009 (in Chinese)

23. Chan JF-W, Yuan S, Kok K-H et al (2020) A familial cluster of pneumonia associated with the 2019 novel coronavirus indicating person-to-person transmission: a study of a family cluster. Lancet 395:514-523

24. Chen N, Zhou M, Dong X et al (2020) Epidemiological and clinical characteristics of 99 cases of 2019 novel coronavirus pneumonia in Wuhan, China: a descriptive study. Lancet 395:507-513

25. Zhang JJ, Dong X, Cao YY et al (2020) Clinical characteristics of 140 patients infected with SARS-CoV-2 in Wuhan, China. Allergy. https://doi.org/10.1111/all.14238

26. Wang $\mathrm{D}, \mathrm{Hu} \mathrm{B}, \mathrm{Hu} \mathrm{C}$ et al (2020) Clinical characteristics of 138 hospitalized patients with 2019 novel coronavirus-infected pneumonia in Wuhan, China. JAMA. https://doi.org/10.1001/jama.2020.1585

27. Wu J, Wu X, Zeng W et al (2020) Chest CT findings in patients with corona virus disease 2019 and its relationship with clinical features. Invest Radiol. https://doi.org/10.1097/rli.0000000000000670

28. Xie X, Zhong Z, Zhao W, Zheng C, Wang F, Liu J (2020) Chest CT for typical 2019-nCoV pneumonia: relationship to negative RTPCR testing. Radiology. https://doi.org/10.1148/radiol.2020200343
29. Fang Y, Zhang H, Xie J et al (2020) Sensitivity of chest CT for COVID-19: comparison to RT-PCR. Radiology. https://doi.org/10. 1148/radiol.2020200432

30. Ma H, Shao J, Wang Y et al (2020) High resolution CT features of novel coronavirus pneumonia in children. Zhonghua Fang She Xue Za Zhi 54:E002 (in Chinese)

31. Ma Y, Xia S, Wang M, Zhang S, Du W, Chen Q (2020) Clinical features of children with SARS-CoV-2 infection: an analysis of 115 cases. Chin J Contemp Pediatr 22:1-4 (in Chinese)

32. Wang D, Ju XL, Xie F et al (2020) Clinical analysis of 31 cases of 2019 novel coronavirus infection in children from six provinces (autonomous region) of northern China. Zhonghua Er Ke Za Zhi 58:E011 (in Chinese)

33. Xia W, Shao J, Guo Y, Peng X, Li Z, Hu D (2020) Clinical and CT features in pediatric patients with COVID-19 infection: different points from adults. Pediatr Pulmonol. https://doi.org/10.1002/ppul.24718

34. Zhou Y, Yang GD, Feng K et al (2020) Clinical features and chest CT findings of coronavirus disease 2019 in infants and young children. Zhongguo Dang Dai Er Ke Za Zhi 22:215-220 (in Chinese)

35. Zhong Z, Xie X, Huang W, Zhao W, Yu Q, Liu J (2020) Chest CT findings and clinical features of coronavirus disease 2019 in children. Zhong Nan Da Xue Xue Bao Yi Xue Ban. https://doi.org/10. 11817/j.issn.1672-7347.2020.200206

36. Sun D, Li H, Lu XX et al (2020) Clinical features of severe pediatric patients with coronavirus disease 2019 in Wuhan: a single center's observational study. World J Pediatr. https://doi.org/10.1007/ s12519-020-00354-4

37. Li HJ, Liu SY, Xu HB, Cheng JL (2020) Guideline for medical imaging in auxiliary diagnosis of coronavirus disease 2019. Chin J Med Imaging Technol 36:1-11 (in Chinese)

38. Liu M, Wan X, Tu XY et al (2020) Family cluster of child SARS-CoV-2 infections: a case report. Wuhan Da Xue Xue Bao. https://doi.org/10.14188/j.1671-8852.2020.0078 (in Chinese)

39. Zhang GX, Zhang AM, Huang L et al (2020) Twin girls infected with SARS-CoV-2. Zhongguo Dang Dai Er Ke Za Zhi 22:221-225 (in Chinese)

40. Zhang Y, Lin D, Xiao M et al (2020) 2019-novel coronavirus infection in a three-month-old baby. Zhonghua Er Ke Za Zhi 58:182184 (in Chinese)

41. Zhao R, Shen X, Xu K, Sheng J (2020) One case report of pediatric infection with COVID-19. Zhejiang Med J. https://doi.org/10. 12056/j.issn.1006-2785.2020.42.3.2020-337 (in Chinese)

42. Cui Y, Tian M, Huang D et al (2020) A 55-day-old female infant infected with COVID 19: presenting with pneumonia, liver injury, and heart damage. J Infect Dis. https://doi.org/10.1093/infdis/jiaa113

43. Ji LN, Chao S, Wang YJ et al (2020) Clinical features of pediatric patients with COVID-19: a report of two family cluster cases. World J Pediatr. https://doi.org/10.1007/s12519-020-00356-2

44. Lou XX, Shi CX, Zhou CC, Tian YS (2020) Three children who recovered from novel coronavirus 2019 pneumonia. J Paediatr Child Health. https://doi.org/10.1111/jpc.14871

45. Wang S, Guo L, Chen L et al (2020) A case report of neonatal COVID-19 infection in China. Clin Infect Dis. https://doi.org/10. 1093/cid/ciaa225

46. National Health Commission of the People's Republic of China, National Administration of Traditional Chinese Medicine (2020) Diagnosis and Treatment Protocol for Novel Coronavirus Pneumonia (Trial Version 7). National Health Commission of the People's Republic of China, National Administration of Traditional Chinese Medicine, China. Available via http://www.nhc.gov.cn/ xcs/zhengcwj/202003/46c9294a7dfe4cef80dc7f5912eb1989. shtml. Accessed 4 Mar 2020 (in Chinese)

Publisher's note Springer Nature remains neutral with regard to jurisdictional claims in published maps and institutional affiliations. 